\title{
Apoptosis and proliferation in lungs of ventilated and oxygen-treated preterm infants
}

\author{
M. May*, P. Ströbel", T. Preisshofen\#, S. Seidenspinner*, A. Marx\#, C.P. Speer*
}

Apoptosis and proliferation in lungs of ventilated and oxygen-treated preterm infants. M. May, P. Ströbel, T. Preisshofen, S. Seidenspinner, A. Marx, C.P. Speer. (C)ES Journals Ltd 2004.

ABSTRACT: Apoptosis and proliferation and the effect of exogenous surfactant on these processes were investigated in the lungs of mechanically ventilated/oxygen-treated preterm infants with respiratory distress syndrome and stillborn foetuses.

Apoptotic and proliferation indices were determined in lung tissue sections from 27 ventilated/oxygen-treated preterm infants and 29 stillborn foetuses. The effect of exogenous surfactant on apoptosis and proliferation was studied in 16 ventilated preterm infants; 11 untreated infants served as control. Apoptotic and proliferating cells were identified by double labelling combining terminal deoxynucleotidyltransferasemediated deoxyuridine triphosphate nick end-labelling or Ki-67 with cell marker proteins. Pathways to cell death were explored by immunolabelling of cleaved caspases$3,-8$ and -9 .

In the lungs of ventilated/oxygen-treated preterm infants, the numbers of apoptotic and proliferating cells increased significantly compared to the respective numbers in the lungs of stillborn foetuses. Apoptosis was detected in alveolar epithelial cells, whereas epithelial, endothelial and smooth muscle cells proliferated. Surfactant treatment reduced apoptosis induced by ventilation/oxygen-treatment; however, the decrease was not significant. Caspases-8 and $\mathbf{- 9}$ do not contribute to ventilation-induced apoptosis, whereas caspase- 3 is involved.

In conclusion, ventilation/oxygen-treatment induces epithelial cell apoptosis and proliferation of epithelial, endothelial and smooth muscle cells in the lungs of preterm infants.

Eur Respir J 2004; 23: 113-121.
*University Children's Hospital and ${ }^{\#}$ Dept of Pathology, University of Würzburg, Würzburg, Germany.

Correspondence: C.P. Speer

University Children's Hospital

Würzburg

Josef-Schneider-Strasse 2

97080 Würzburg

Germany

Fax: 4993120127833

E-mail: speer_c@klinik.uni-wuerzburg.de

Keywords: Cell death

hyperoxia

pulmonary surfactant

signal transduction

Received: April 72003

Accepted after revision: July 292003
Apoptosis and proliferation are involved in several processes during embryonal and foetal life and play an important role in normal cell turnover and tissue development.

Although they have been implicated as mechanisms of obtaining correct cell number, they are involved in pathological processes. In the lung, these processes can result from oxygen injury caused by high oxygen concentrations. However, oxygen therapy is often necessary for patients suffering from pulmonary diseases. This is particularly true for preterm infants and neonates suffering from respiratory distress syndrome (RDS), who require supraphysiological concentrations of oxygen to maintain adequate blood oxygen tensions.

In animal models, it has been demonstrated that apoptosis is significantly increased in lungs exposed to hyperoxia [1-3]. In addition, it could be shown that hyperoxia induces increased expression of genes encoding apoptosis-promoting proteins [1, 2]. Proliferation, however, ceases as a consequence of hyperoxia in cell culture experiments with alveolar epithelial cells, fibroblasts and tracheal smooth muscle cells [4-6].

Exogenous surfactant therapy remains an established treatment of RDS. Recently, it has become increasingly obvious that exogenous surfactant preparations have significant effects on cell physiology and inflammatory processes in the lung comprising suppression of pro-inflammatory cytokines, superoxide production and regulation of the pulmonary host defence [7, 8]. However, nothing is known about the influence of surfactant on apoptosis and proliferation of lung tissue cells in hyperoxia-damaged lung.
Although apoptosis and proliferation obviously play an important role in the development and hyperoxic damage of lung tissue, investigations have merely been made on animal models, and only one recent study considers apoptosis in several organs of ventilated preterm infants with RDS [9]. The work in the present report was conducted to examine apoptosis and proliferation, particularly in the lungs of human stillborn foetuses and the lung tissues of preterm infants with RDS exposed to mechanical ventilation and elevated oxygen concentrations. The occurrence of both processes as integral parts of lung development and the effects of ventilation at elevated oxygen concentrations on apoptosis and proliferation were studied. In these hyperoxia-damaged premature human lungs, the possible influence of surfactant on apoptosis and proliferation was also investigated. Additionally, it was investigated whether caspases-3, -8 or -9 are activated in the signalling cascade leading to apoptosis.

\section{Material and methods}

\section{Study population}

The reports from all infant autopsy procedures performed in the Dept of Pathology (University of Würzburg, Würzburg, Germany) during 1985-2000 were reviewed.

Foetuses and preterm infants delivered at 22-36 weeks of gestation without pulmonary or urogenital malformation 
were selected for the study if they showed no pneumonia, chorioamnionitis, inflammation of the umbilical cord or placentitis; 159 cases fulfilled these criteria. The corresponding lung tissue autopsy samples had been routinely formalinfixed and paraffin embedded. Sections had been cut at a thickness of $4 \mu \mathrm{m}$ and stained with haematoxylin and eosin. They were examined microscopically and only nonautolytic and well-preserved material was selected for the present study. Fifty-six subjects were chosen and the study population divided into two groups. One group comprised 29 stillborn foetuses aged $22-36$ weeks of gestation (mean \pm SEM $28.0 \pm 0.8$ weeks) and the other 27 infants live born at 23-36 weeks of gestation ( $27.9 \pm 0.7$ weeks) with RDS, who had received ventilatory support and supplemental oxygen. Ventilator records were reviewed and the mean daily inspiratory oxygen fraction and mean airway pressure were determined and averaged over the duration of ventilator treatment.

In order to investigate whether changes in numbers of apoptotic and proliferating cells depend on the duration of ventilation at elevated oxygen concentrations, preterm infants with RDS were divided into three groups, infants ventilated for $0-1$ days, 1-3 days and $>3$ days, respectively. In addition, surfactant treatment and nontreatment were considered in mechanically ventilated/oxygen-treated preterm infants with RDS.

Clinical details of the infants studied are given in table 1 .

\section{Terminal deoxynucleotidyltransferase-mediated deoxyuridine triphosphate nick end-labelling assay}

Deoxyribonucleic acid (DNA) fragmentation associated with apoptosis was detected by terminal deoxynucleotidyltransferasemediated deoxyuridine triphosphate nick end-labelling (TUNEL), using an Apopdetect kit (Qbiogene, Heidelberg, Germany) according to the manufacturer's instructions. Peroxidase was visualised using $20 \mathrm{mg} \cdot \mathrm{L}^{-1} 3,3^{\prime}$-diaminobenzidine tetrahydrochloride in $0.05 \mathrm{M}$ tris-(hydroxymethyl)aminomethane (Tris) ( $\mathrm{pH} 7.6$ ) containing $0.01 \%$ hydrogen peroxide. The specimens were rinsed in water and counterstained with $1 \mathrm{~g} \cdot \mathrm{L}^{-1}$ Mayer's haematoxylin solution. Negative controls were processed with labelled deoxyuridine triphosphate in the absence of terminal deoxynucleotidyltransferase.

\section{Ki-67 immunohistochemistry}

Immunocytochemical demonstration of $\mathrm{Ki}-67$ was performed employing a rabbit polyclonal antibody directed against Ki-67 (Dako, Hamburg, Germany) diluted 1:50 in $0.25 \mathrm{M}$ phosphate-buffered saline (PBS) ( $\mathrm{pH} \mathrm{7.4)/10 \%}$ foetal calf serum (FCS) and a streptavidin/biotin conjugate kit (Biogenex, San Ramon, CA, USA) with $20 \mathrm{mg} \cdot \mathrm{L}^{-1}$ 3,3'-diaminobenzidine tetrahydrochloride as chromogen, in $0.05 \mathrm{M}$ Tris $(\mathrm{pH} 7.6)$ containing $0.01 \% \mathrm{H}_{2} \mathrm{O}_{2}$. Tissue sections were microwaved six times for 5 min each at $800 \mathrm{~W}$ in $10 \mathrm{mM}$ citrate buffer ( $\mathrm{pH}$ 6.0). Negative controls consisted of samples submitted to primary incubation with PBS/FCS. The sections were counterstained with $1 \mathrm{~g} \cdot \mathrm{L}^{-1}$ Mayer's haematoxylin solution.

\section{Quantification of apoptosis and proliferation}

In order to quantify the extent of apoptosis and proliferation in the lung tissues, four random fields from each tissue section were photographed at 40-fold magnification using a Zeiss Axiophot microscope (Zeiss, Oberkochen, Germany)

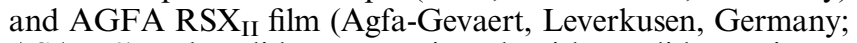
ASA 50). The slides were viewed with a slide projector (Kindermann magic screen IR, Ochsenfurt, Germany) for subsequent counting, which was performed manually. The total number of nuclei and the number of positively stained nuclei in each field were determined; 500-750 nuclei per field, i.e. 2,000-3,000 nuclei per tissue section were counted. The apoptotic index (AI) was calculated as the percentage of the total number of nuclei that were TUNEL-positive or apoptotic, and the proliferation index (PI) as the percentage that were Ki-67-immunostained.

\section{Caspase immunohistochemistry}

For the immunohistochemical detection of cleaved, i.e. activated, caspases- 8 and -9 , the monoclonal antibody $11 \mathrm{G} 10$ (New England Biolabs, Beverly, MA, USA) and a rabbit polyclonal caspase-9 cleavage site (315/316)-specific antibody (Biosource International, Nivelles, Belgium) were used, each diluted 1:50 in 0.25 M PBS ( $\mathrm{pH} 7.4) / 10 \%$ FCS. Although tissue sections submitted to cleaved caspase-9 immunolabelling were not microwaved, tissue sections for the immunohistochemical detection of cleaved caspase- 8 were microwaved twice for $5 \mathrm{~min}$ each in citrate buffer. Immunohistochemistry was performed using the streptavidin/biotin/peroxidase method.

For detection of activated caspase-3, tissue sections were boiled in citrate buffer for $5 \mathrm{~min}$ at $124^{\circ} \mathrm{C}$ and immunostained using activated caspase- 3 antibody (New England Biolabs)

Table 1.-Clinical details of study population

\begin{tabular}{|c|c|c|c|c|c|c|c|}
\hline & \multirow[t]{3}{*}{ Stillborn foetuses } & \multicolumn{6}{|c|}{ Preterm infants ${ }^{\#}$} \\
\hline & & \multirow[t]{2}{*}{ Total } & \multicolumn{3}{|c|}{ Duration of ventilation days } & \multicolumn{2}{|c|}{ Surfactant treatment } \\
\hline & & & $0-1$ & $1-3$ & $>3$ & Yes & No \\
\hline Subjects n & 29 & 27 & 15 & 5 & 7 & 16 & 11 \\
\hline Gestational age weeks & $28.0 \pm 0.8$ & $27.9 \pm 0.7$ & $27.5 \pm 0.9$ & $26.2 \pm 1.3$ & $30.1 \pm 1.4$ & $27.0 \pm 0.9$ & $28.6 \pm 1.0$ \\
\hline Birthweight $\mathrm{g}$ & $1006 \pm 114$ & $1138 \pm 106$ & $1064 \pm 117$ & $876 \pm 247$ & $1614 \pm 220$ & $1007 \pm 172$ & $1285 \pm 135$ \\
\hline Length $\mathrm{cm}$ & $34.8 \pm 1.5$ & $38.0 \pm 1.1$ & $37.6 \pm 1.4$ & $35.6 \pm 3.0$ & $41.4 \pm 1.0$ & $38.0 \pm 1.6$ & $38.4 \pm 1.4$ \\
\hline Mean $P$ aw $\mathrm{mmHg}$ & - & $21.9 \pm 1.9$ & $23.8 \pm 2.8$ & $19.9 \pm 3.4$ & $19.3 \pm 3.0$ & $18.8 \pm 2.2$ & $24.1 \pm 2.6$ \\
\hline Mean FI, $\mathrm{O}_{2}$ & - & $0.7 \pm 0.1$ & $0.9 \pm 0.1$ & $0.6 \pm 0.1$ & $0.5 \pm 0.1$ & $0.7 \pm 0.1$ & $0.8 \pm 0.1$ \\
\hline Males/females n & $11 / 18$ & $16 / 11$ & $8 / 7$ & $4 / 1$ & $4 / 3$ & $10 / 6$ & $6 / 5$ \\
\hline
\end{tabular}

Data are presented as mean \pm SEM. The median duration of ventilation of children ventilated for $>3$ days was 6.8 days; $P$ aw: airway pressure during artificial ventilation; FI, $\mathrm{O}_{2}$ : inspiratory oxygen fraction during artificial ventilation. Stillborn foetuses died because of abortion ( $\mathrm{n}=18$ ), intrauterine foetal death $(n=2)$, ablatio placentae $(n=4)$, intrauterine asphyxia $(n=4)$ and circulatory failure $(n=1)$. Causes of death of ventilated preterm infants with respiratory distress syndrome (RDS) were respiratory insufficiency $(n=14)$, circulatory failure $(n=11)$ and cerebral haemorrhage $(n=2)$. \#: ventilated/oxygen-treated due to RDS; $(1 \mathrm{mmHg}=0.133 \mathrm{kPa})$. 
diluted 1:20 and the Histostain Plus Kit (Zytomed, Berlin, Germany).

\section{Double immunolabelling}

Double immunolabelling was performed, combining immunoperoxidase with 3,3'-diaminobenzidine tetrahydrochloride as chromophore for the detection of TUNELpositive nuclei and Ki-67, respectively, and immunoalkaline phosphatase with Fast Blue BBN diazonium salt and naphthol AS-MX phosphate as substrate for the detection of cytokeratin 18, CD34 or muscle actin. Peroxidase was developed using $20 \mathrm{mg} \cdot \mathrm{L}^{-1} 3,3^{\prime}$-diaminobenzidine tetrahydrochloride in $0.05 \mathrm{M}$ Tris ( $\mathrm{pH}$ 7.6) containing $0.01 \% \mathrm{H}_{2} \mathrm{O}_{2}$ and alkaline phosphatase activity was visualised wih $50 \mathrm{~mL}$ $1 \mathrm{mg} \cdot \mathrm{mL}^{-1}$ Fast Blue BNN diazonium salt in Tris ( $\mathrm{pH}$ 7.4) containing $1 \mathrm{~mL} N, N$-dimethylformamide, $10 \mathrm{mg}$ naphthol AS-MX phosphate and $25 \mathrm{mg}$ levamisole for $10 \mathrm{~min}$ to give blue deposits. Mouse monoclonal antibodies directed against cytokeratin 18 (clone DC 10), CD34 (clone QBEnd 10) and muscle actin (clone HHF35; all Dako) were diluted 1:50 in 0.25 M PBS (pH 7.4)/10\% FCS and incubated overnight. The antibodies were detected using a streptavidin/biotin conjugate kit (Biogenex, Hamburg, Germany).

\section{Statistical analysis}

One-way analysis of variance was used to compare the differences in AI and PI of stillborn foetuses at different gestational ages. The AIs and PIs of stillborn foetuses versus mechanically ventilated/oxygen-treated preterm infants and of the surfactant treated/nontreated groups were compared using the Mann-Whitney U-test. The differences in AI and PI for different durations of mechanical ventilation/oxygen treatment were analysed using the Kruskal-Wallis test followed by pairwise comparisons using the Mann-Whitney U-test. A p-value of $<0.05$ was considered significant. Data are presented as medians and interquartile ranges.

\section{Results}

\section{Apoptosis and proliferation in human foetal lung (stillborn foetuses)}

Lung sections from human stillborn foetuses of ages ranging 22-36 weeks of gestation were examined for apoptotic cells, which were visualised in situ using the TUNEL assay. It cannot be excluded that necrotic cells also contained TUNEL stainable concentrations of $3^{\prime}$-hydroxyl DNA ends. However, TUNEL-positive cells showed morphological
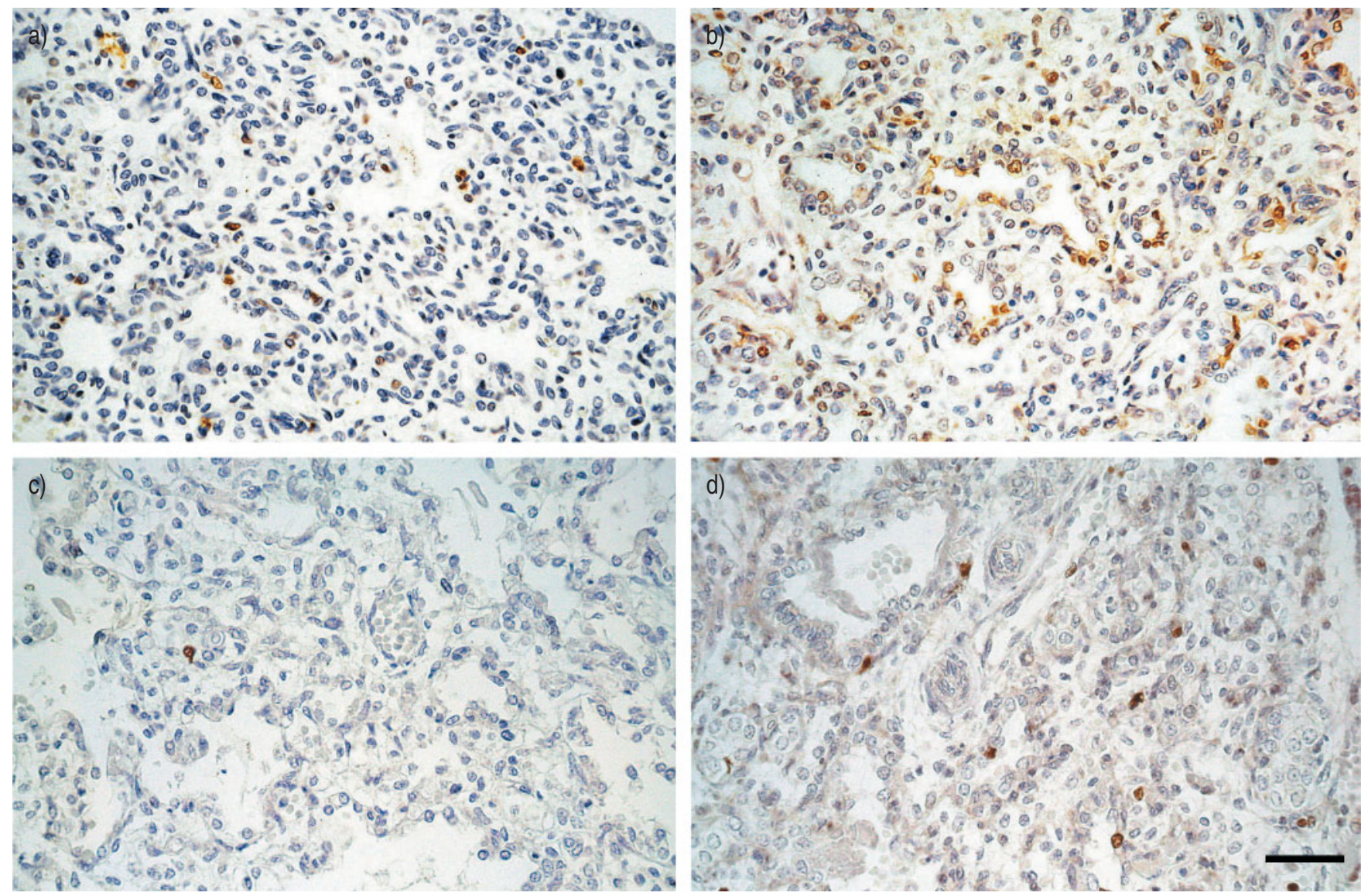

Fig. 1.-Apoptotic cells in the lungs of stillborn foetuses and mechanically ventilated/oxygen-treated preterm infants with respiratory distress syndrome. Terminal deoxynucleotidyltransferase-mediated deoxyuridine triphosphate nick end-labelling (TUNEL) of a representative section of the lung of: a) a stillborn foetus aged 28 weeks of gestation; TUNEL-positive nuclei are sparse; and b) the lung of a preterm infant of 26 weeks of gestation, ventilated at elevated oxygen concentrations for $10 \mathrm{~h}$; TUNEL-positive nuclei are more numerous compared with the lung from the stillborn foetus. Anti-Ki-67 immunohistochemical staining of a representative section of the lung of: c) a stillborn infant aged 30 weeks of gestation; there are few Ki-67-positive cells; and d) a preterm infant aged 28 weeks of gestation, who was ventilated at elevated oxygen levels for $13 \mathrm{~h}$; Ki-67-positive cells are more numerous than in the lung of the stillborn foetus. Internal scale bar=50 $\mu \mathrm{m}$. 
features of apoptosis such as pyknotic nuclei and cell shrinkage. Additionally, immunohistochemical staining of activated caspase-3, an important executioner caspase of the apoptotic signalling pathway, was performed. This confirmed the occurrence of apoptotic cells and the results of the TUNEL assays.

Quantification of TUNEL-positive nuclei revealed no significant changes in AI in foetal lungs of gestational age 22-36 weeks. The median AI remained constant at 0.8 (0.4-1.5).

The same lung samples were subjected to Ki-67 immunohistochemistry to determine the degree of cellular proliferation. The median PI remained constant at 1.8 (1.4-3.2) during this period of development.

Apoptosis and proliferation in the lungs of preterm infants with respiratory distress syndrome treated with ventilation at elevated oxygen concentrations

In lung tissue sections from preterm infants with RDS aged 23-36 weeks of gestation who required mechanical ventilation and supplemental oxygen, considerably more TUNELpositive nuclei were demonstrated than in lung tissue sections from stillborn foetuses (fig. 1a and b). This result was confirmed by anti-activated caspase- 3 immunohistochemistry, which also revealed more apoptotic cells in lung tissue sections from mechanically ventilated and oxygen-treated preterm infants than in those from stillborn foetuses (fig. 2).

The median percentage of cells undergoing apoptosis in the lungs of ventilated/oxygen-treated RDS infants was six-fold higher than the median AI of lungs of stillborn foetuses (ventilated/oxygen-treated infants 4.8 (2.4-10.4), stillborn foetuses $0.8(0.4-1.5) ; \mathrm{p}<0.001)$ (fig. 3a).

As with TUNEL-positive cells, the number of proliferating (Ki-67-positive) cells was raised in the lungs of preterm infants with RDS in association with mechanical ventilation/ oxygen treatment (fig. 1c and d). The median PI was 3.9 (1.9-6.7) in this group and $1.8(1.4-3.2)$ in the stillborn foetus group $(\mathrm{p}<0.01)$ (fig. $3 b)$.

Levels of apoptosis and proliferation are dependent on the duration of mechanical ventilation at elevated oxygen concentrations

Compared with the AI of lungs from stillborn foetuses, the percentage of TUNEL-positive cells in lungs from preterm infants with RDS, who were ventilated at elevated oxygen concentrations, increased significantly after $<1$ day of ventilation, as well as after 1-3 days and $>3$ days of ventilation (stillborn foetuses $0.8(0.4-1.5)$, ventilation $<1$ day $2.8(1.0-8.0), 1-3$ days $3.3(2.2-7.7),>3$ days 13.3 (7.5-15.5); $\mathrm{p}<0.001)$ (fig. 4a).

The influence of the duration of mechanical ventilation at elevated oxygen concentrations on PI in the lungs of preterm infants with RDS was also evaluated. The median PI increased with prolonged ventilation (stillborn foetuses 1.8 (1.4-3.2), ventilation $<1$ day 2.8 (1.5-4.6), 1-3 days 3.7 (2.4-5.0), >3 days 6.9 (6.0-10.7); $\mathrm{p}<0.001)$ (fig. 4b).

\section{Identification of apoptotic and proliferating cells}

Double staining of the lungs of stillborn foetuses revealed that, in the stage of lung development occurring at 22-25 weeks of gestation, the sparse TUNEL-positive nuclei were preferentially localised in mesenchymal and endothelial cells (fig. 5a and b), whereas, in the lungs of older stillborn foetuses

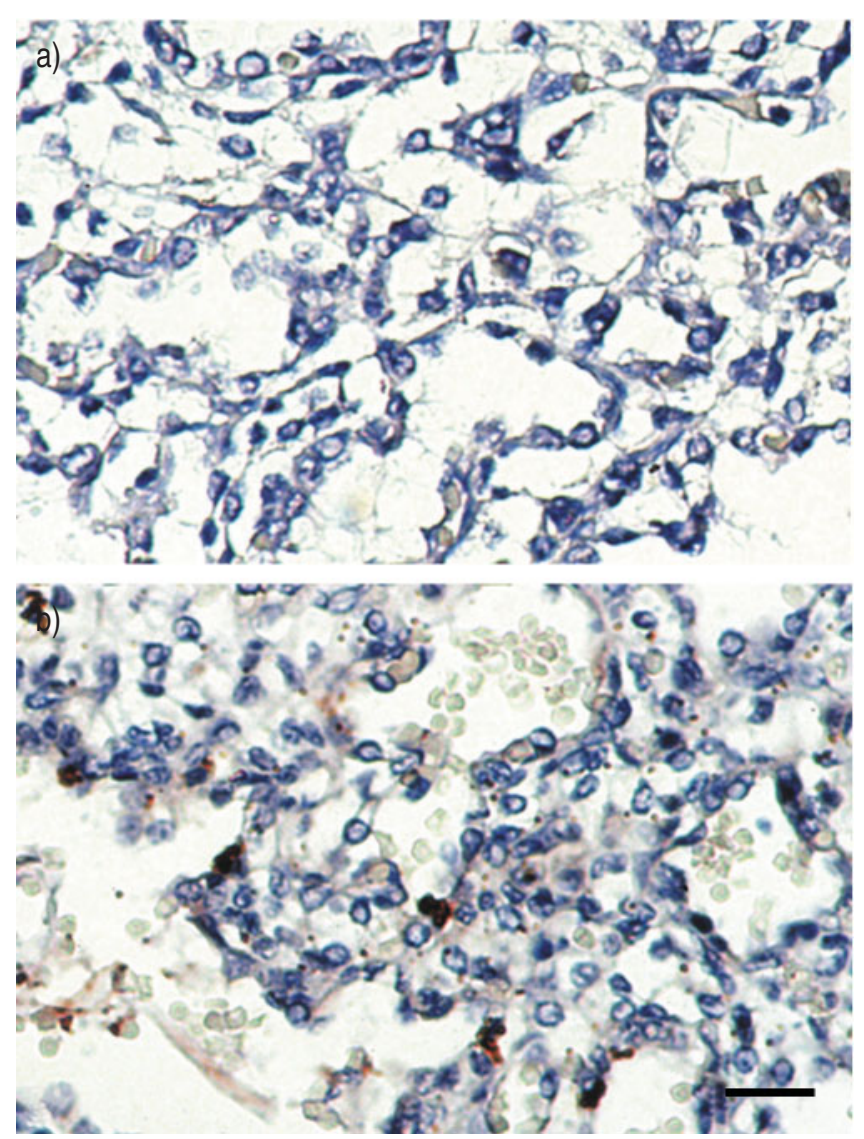

Fig. 2.-Representative lung tissue sections from: a) a stillborn foetus aged 29 weeks of gestation; and b) a preterm infant with respiratory distress syndrome aged 30 weeks of gestation and ventilated at elevated oxygen concentrations for $11 \mathrm{~h}$ showing immunohistochemical staining of cleaved, i.e. activated, caspase-3 (brown). Haematoxylinstained nuclei are blue. Immunopositive cells are more numerous in the lung of the preterm infant than in that of the stillborn foetus and mainly localised in the alveolar epithelium. Internal scale bar $=25 \mu \mathrm{m}$.

(26-36 weeks of gestation), apoptotic nuclei were detected mainly in alveolar epithelial cells (fig. 5c).

In lung specimens from preterm infants with RDS exposed to mechanical ventilation at elevated oxygen concentrations, double labelling revealed that mainly cytokeratin 18-positive epithelial cells were apoptotic (fig. 6a and b). By contrast, only few TUNEL-positive nuclei were present in CD34-positive endothelial or smooth muscle $\alpha$-actin-positive muscle cells.

Double labelling performed to identify proliferating cell types revealed that there was no preferred cell type, neither in lung tissues from stillborn foetuses nor in mechanically ventilated/oxygen-treated lung specimens.

\section{Influence of surfactant on apoptosis and proliferation}

The AIs as well as the PIs of ventilated/oxygen-treated preterm infants with RDS who received surfactant were compared with those who did not.

The median AI of the lungs of ventilated/oxygen-treated infants with RDS who received surfactant was less than half that found in patients not treated with surfactant (surfactant 3.3 (2.8-7.5), no surfactant 7.4 (1.6-11.7)) (fig. 7). However, statistical analysis revealed that the difference in AI between the experimental groups was not significant.

The median PI of the lungs of mechanically ventilated/ oxygen-treated preterm infants with RDS in the presence and absence of surfactant therapy was identical (surfactant 3.9 

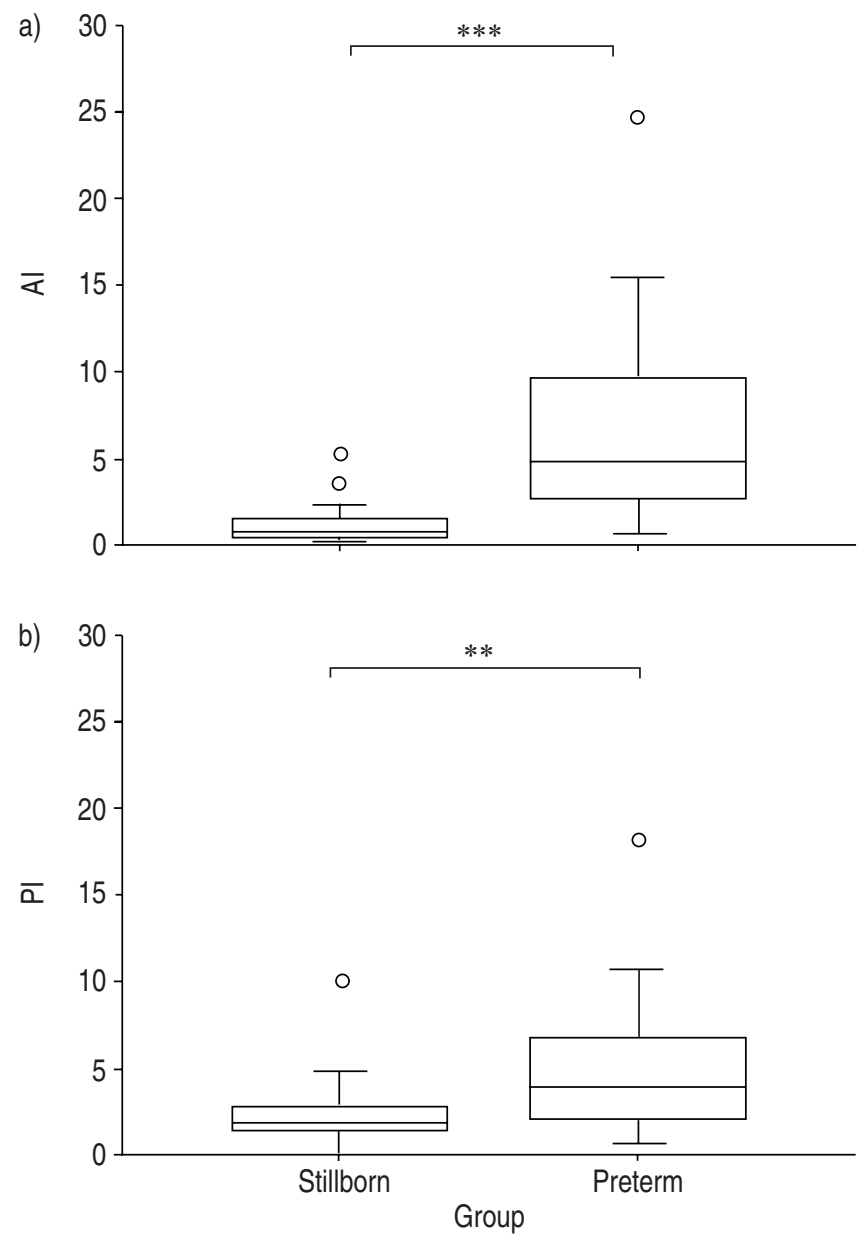

Fig. 3.-Boxplot showing effect of mechanical ventilation at elevated oxygen concentrations on: a) apoptotic (AI); and b) proliferation indices $(\mathrm{PI})$ in the lungs of preterm infants with respiratory distress syndrome (RDS; $n=27)$ compared to stillborn foetuses $(n=29)$. Data are presented as median and interquartile range; the vertical bars represent the 10th and 90th percentiles ( $\bigcirc$ : outliers). The median AI was six-fold and the median PI two-fold higher in the lungs of ventilated preterm infants with RDS than in those of stillborn foetuses. ${ }^{*}: \mathrm{p}<0.01 ; * *: \mathrm{p}<0.001$

(1.9-7.9), no surfactant 3.8 (1.9-6.5)). Therefore, proliferation was obviously not influenced by surfactant treatment in the lungs of mechanically ventilated/oxygen-treated preterm infants with RDS.

\section{Pathways leading to apoptosis in mechanically ventilatedl oxygen-treated lungs}

In order to explore the biochemical pathways leading to cell death in the lungs of stillborn foetuses and ventilated/oxygentreated preterm infants with RDS, immunolabelling of cleaved caspases-3, -8 and -9 was performed.

In the lung tissues of stillborn foetuses, cleaved caspases- 8 and -9 were observed occasionally, in both epithelial and interstitial cells. After ventilation at elevated oxygen concentrations, there was no change in either the extent or distribution of cleaved caspase-8- or -9-immunopositive cells.

However, in the lung tissues of ventilated/oxygen-treated preterm infants with RDS, a considerable increase in the amount of activated caspase- 3 was detectable compared with the lung tissues of stillborn foetuses. As shown in figure $2 b$,
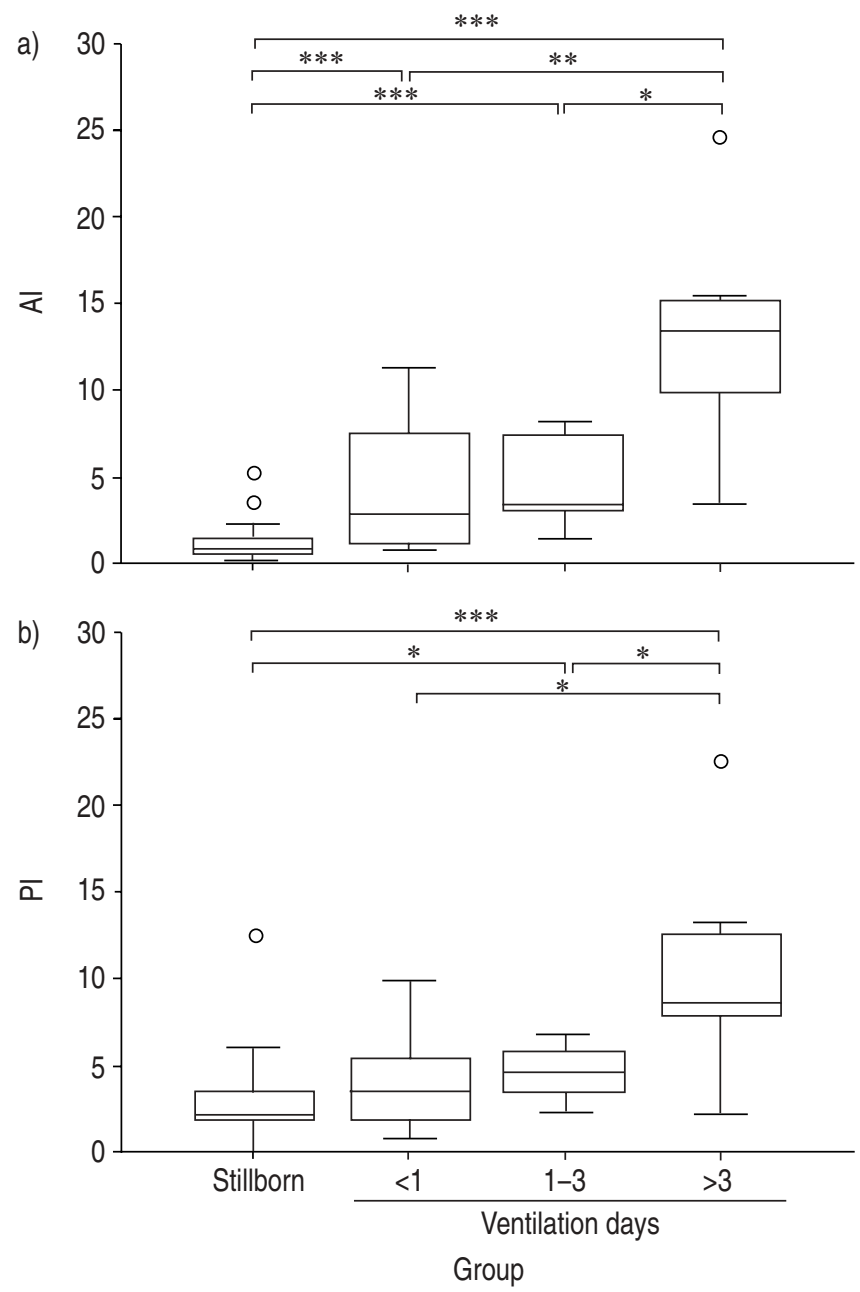

Fig. 4. - Boxplot showing effect of duration of mechanical ventilation at elevated oxygen concentrations on: a) apoptotic (AI); and b) proliferation indices (PI) in the lungs of preterm infants with respiratory distress syndrome (RDS) divided into three groups according to duration of ventilation $(<1$ day: $n=15 ; 1-3$ days: $n=5 ;>3$ days: $n=7)$ compared to stillborn foetuses $(n=29)$. Data are presented as median and interquartile range; the vertical bars represent the 10th and 90th percentiles $(\bigcirc$ : outliers). AI and PI increased significantly with prolonged mechanical ventilation at elevated oxygen concentrations. *: p<0.05; **: $p<0.01 ; * * *: p<0.001$.

activated caspase-3-immunopositive cells were numerous and mainly alveolar epithelial cells. On the contrary, in the lungs of stillborn foetuses, activated caspase- 3 was merely detectable immunohistochemically (fig. 2a).

\section{Discussion}

In the present study, it was demonstrated that the numbers of apoptotic and proliferating cells were increased significantly in lungs from preterm infants with RDS as a consequence of mechanical ventilation at elevated oxygen levels. Exogenous surfactant had no influence on cellular proliferation, but might have an antiapoptotic effect. Initiator caspases- 8 and -9 of apoptosis signalling pathways did not contribute to apoptosis induced by mechanical ventilation at elevated oxygen concentrations, whereas caspase- 3 was involved.

It should be borne in mind that the clinical treatment of preterm infants is a complex process harmonised with the 

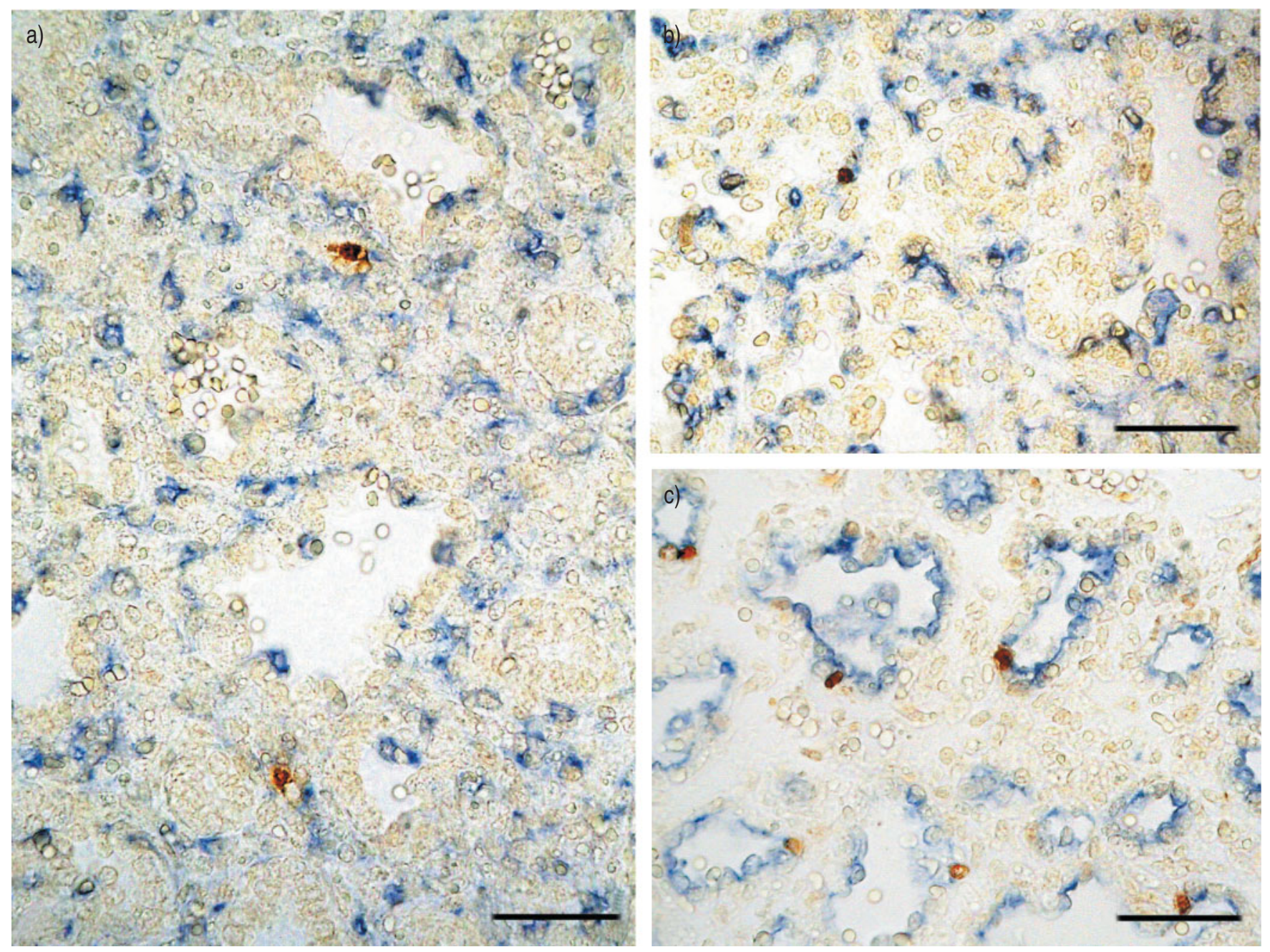

Fig. 5.-Double labelling experiments in the lungs of stillborn foetuses of different age using terminal deoxynucleotidyltransferase-mediated deoxyuridine triphosphate nick end-labelling (TUNEL) and antibodies directed against cytokeratin 18 (epithelial cell marker) or CD34 (endothelial cell marker). TUNEL-positive cells are brown; cell marker proteins are blue. a, b) TUNEL- and CD34-positive endothelial cells in the lung of a stillborn foetus at 23 weeks of gestation; and c) TUNEL- and cytokeratin 18-positive alveolar epithelial cells in the lung of a stillborn foetus at 28 weeks of gestation. The localisation of the sparse TUNEL-positive cells shifts from being more mesenchymal/endothelial ( $\mathrm{a}$ and $\mathrm{b}$ ) to being preferentially epithelial (c). Internal scale bars $=50 \mu \mathrm{m}$.

individual needs of the patients. Therefore, a certain heterogeneity of the groups examined could not be avoided. Moreover, mechanical ventilation with supplemental oxygen is associated with hyperoxia as well as elevated airway pressure and mechanical stretch, and each of these factors may exert different effects on apoptosis and proliferation. The mechanisms activating apoptosis and proliferation by physical forces remain to be investigated. Apoptosis following cyclic stretch has been shown in type II epithelial cells and fibroblasts [10, 11]. Proliferation of rat lung fibroblasts, however, is inhibited by cyclic mechanical stretch [12], whereas, in $\alpha$-smooth muscle cells, strain increases proliferation [13]. Cellular reactions to hyperoxia in the lung have been investigated in animal models, and there exposure to hyperoxia has been shown to induce apoptosis and proliferation $[1-3,11]$. The present study of the effects of mechanical ventilation at elevated oxygen concentrations on apoptosis and proliferation in the lungs of preterm infants with RDS led to results which are in good agreement with the findings in animal models of hyperoxia.

The present study clearly shows that proliferation and apoptosis in human foetal lungs are normal processes during the canalicular and saccular stages of lung development.
Proliferation was demonstrated in epithelial, endothelial and muscle cells, a finding in agreement with previous investigations [14]. The PI was low and did not vary significantly during weeks $22-36$ of gestation. In contrast, other investigators have described a gradual decrease in proliferation during late gestation in human lungs [15].

The apoptotic activity of the lungs of human stillborn foetuses of the examined developmental period was also low and did not change. This result is in good agreement with studies that have described low and constant AIs in the developing rat [16] and human foetal lungs [15]. In accordance with results obtained from studies with rabbits [17], a shift was observed from more mesenchymal and endothelial to predominantly epithelial cell apoptosis at the transition from the canalicular to the terminal saccular stage (26th gestational week). It is possible that the increased epithelial cell apoptosis may be connected to the differentiation of type II and type I alveolar epithelial cells, occurring at this time [18].

The data presented show that the numbers of apoptotic and proliferating cells in human foetal lungs increase significantly with mechanical ventilation at elevated oxygen concentrations. 

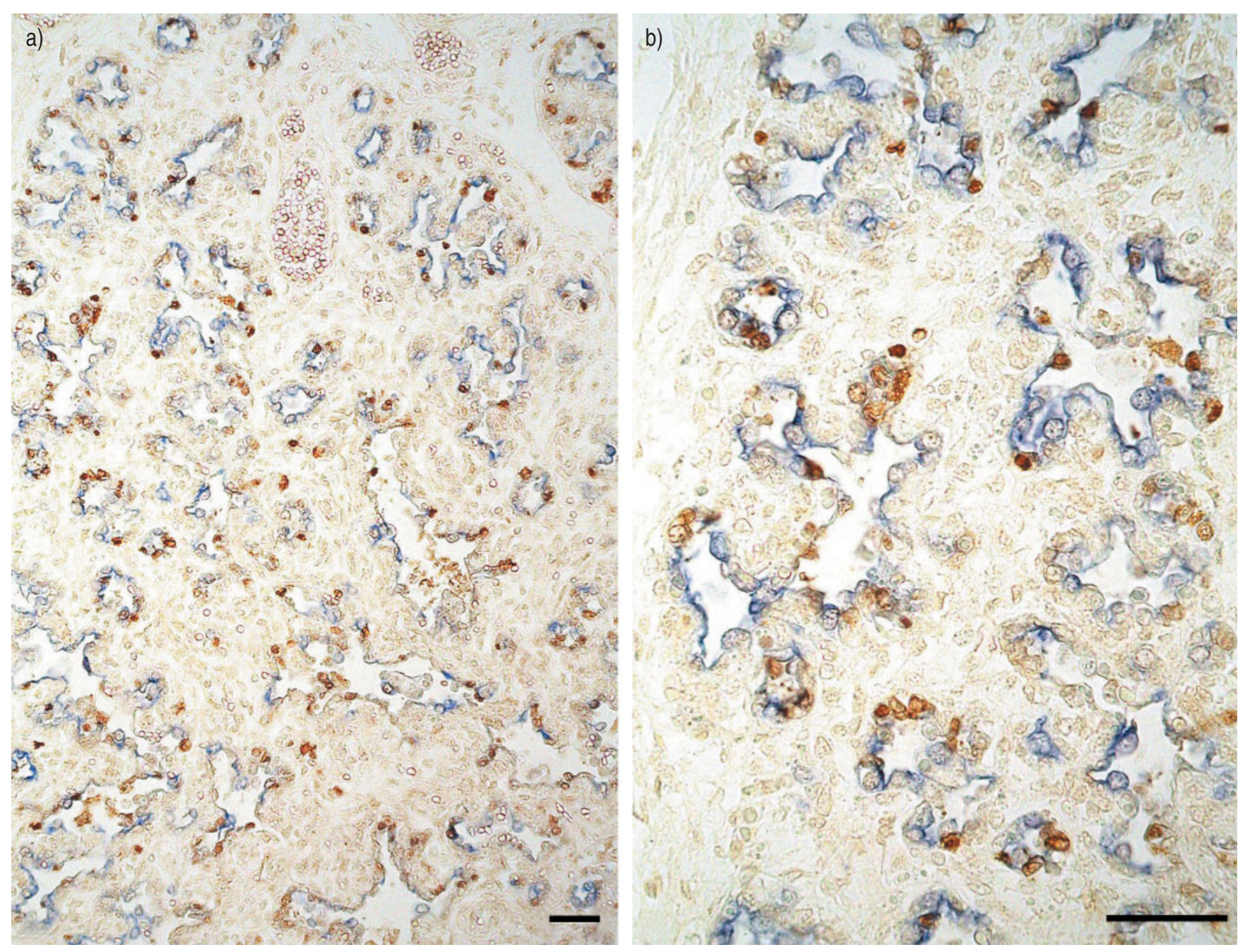

Fig. 6. - a, b) Distribution of apoptotic bodies and epithelial cells found in double labelling experiments using an antibody directed against cytokeratin 18 (epithelial cell marker) and terminal deoxynucleotidyltransferase-mediated deoxyuridine triphosphate nick end-labelling (TUNEL) in a representative lung section from a preterm infant with respiratory distress syndrome at 28 weeks of gestation, who was mechanically ventilated/oxygen-treated for $21 \mathrm{~h}$. TUNEL-positive nuclei are brown; epithelial cells are blue. Most of the labelling is colocalised. Internal scale bars $=50 \mu \mathrm{m}$

The median PI of lungs from preterm infants was increased 2.2-fold. This observation is in contradiction to results obtained in cell culture experiments, in which hyperoxia inhibited the proliferation of fibroblasts [5], smooth muscle cells [4] and epithelial cells [19]. The cessation of proliferation is believed to represent a basic protective mechanism that prevents the replication of damaged template DNA. Molecules such as $\mathrm{p} 21^{\mathrm{WAF} / \mathrm{CIP} 1}$ and $\mathrm{p} 53$, which promote cell cycle arrest, are upregulated during hyperoxia [20] and the messenger ribonucleic acids (mRNAs) encoding the cell cycle proteins histone and thymidine kinase are not translated under hyperoxic conditions [19]. However, in rat lung and in lung explant cultures, under hyperoxic conditions, increased proliferation, especially of alveolar type II cells and fibroblasts, was observed [10, 11]. This indicates that, in contrast to cell culture experiments, tissue cell proliferation is influenced by cell/cell interactions of different cell types. Mitogens such as basic fibroblast growth factor 8 [21], keratinocyte growth factor [22], and insulin-like growth factor-I [23] could stimulate proliferation during recovery. During tissue repair, proliferation of all pulmonary cell types can be detected [24]. In lungs ventilated at elevated oxygen concentrations, no preferentially proliferating cell type could be identified.
The profound increase in apoptosis in the lung tissues of human preterm infants, who received ventilatory and oxygen support, reached a median AI that was six-fold higher than that in stillborn foetuses.

Moreover, the duration of mechanical ventilation/oxygen treatment plays a critical role in pulmonary apoptosis in preterm infants. With increasing duration of mechanical ventilation at elevated oxygen levels, a considerably increasing median percentage of apoptotic cells could be demonstrated. In several hyperoxic animal model systems, the increase in the percentage of cells undergoing apoptosis was likewise time dependent $[1,2]$. This could be due to an accumulation of reactive oxygen species (ROS). Studies performed on alveolar epithelial cell cultures revealed that apoptosis was not induced by pure oxygen but by ROS [25]. These molecules are produced in the mitochondrion by its electron transport system [26], by infiltrating neutrophils and phagocytotic cells [27] and by oxidases [28]. The ROS accumulate in lung tissues with prolonged ventilation at elevated oxygen concentrations. The immaturity of cellular antioxidant systems in the lungs of preterm infants [29] most probably results in deficient clearance of ROS and $\mathrm{H}_{2} \mathrm{O}_{2}$ by antioxidants and prevents effective limitation of the oxidant burst that 


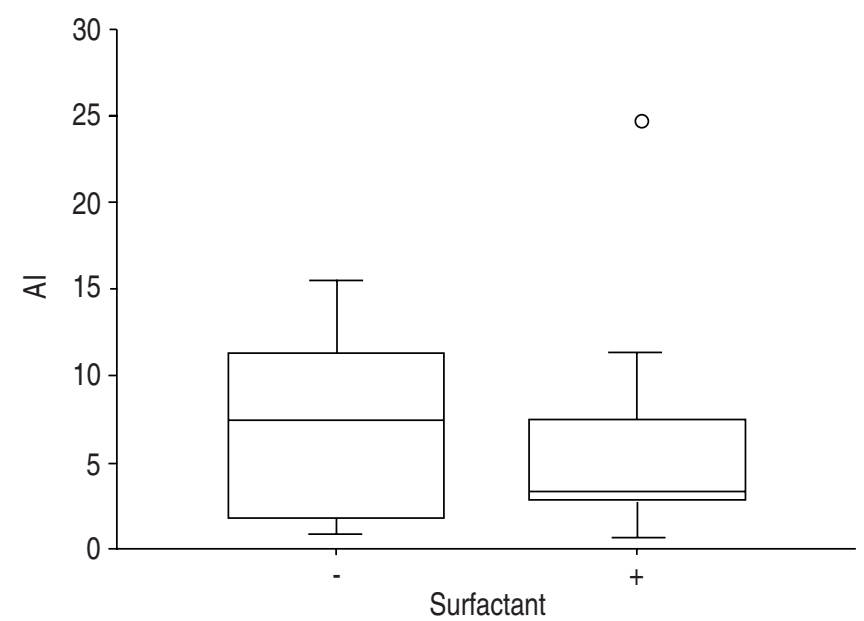

Fig. 7.-Boxplot showing effect of exogenous surfactant on apoptotic index (AI) in the lungs of mechanically ventilated/oxygen-treated preterm infants with respiratory distress syndrome. Data are presented as median and interquartile range; the vertical bars represent the 10th and 90th percentiles ( $\bigcirc$ : outliers). The median AI in the surfactant-treated group $(n=11)$ was less than half that without surfactant treatment $(\mathrm{n}=16)$.

mediates molecular damage leading to an alteration in cell physiology and apoptosis.

In the lungs of mechanically ventilated/oxygen-treated preterm infants with RDS of 23-36 weeks of gestation, apoptosis was preferentially increased in alveolar and bronchiolar epithelial cells. In mice, BARAZZONE et al. [1] found, additionally, increased apoptosis of pulmonary endothelial cells under hyperoxia. Investigating apoptosis in the lungs of mechanically ventilated/oxygen-treated preterm infants with RDS, this finding could not be confirmed. Alveolar epithelial cells serve as a first-line defence against hyperoxic insults and contain antioxidant activity that increases under hyperoxic conditions, as shown for type II pneumocytes [30]. Other studies in the lungs of animals exposed to hyperoxia also revealed that, although all cellular compartments are at risk of oxidative damage, alveolar and bronchiolar epithelial cells are the primary targets of oxygen toxicity and undergo apoptosis [3].

Since various studies in animal models and cell culture have emphasised the physiological and immunomodulatory effects of pulmonary surfactant [7,8], the effect of exogenous surfactant on apoptosis and proliferation in ventilated/ oxygen-treated lungs was of interest. Previously, it had been shown that surfactant inhibited proliferation in fibroblast cultures [8], and surfactant lipids also cause suppression of mitogen-induced lymphocyte proliferation [31]. However, in the lungs of preterm infants with RDS exposed to mechanical ventilation and supplemental oxygen, exogenous surfactant has no effect on proliferation.

Concerning apoptosis, only a trend was observed towards a protective effect of exogenous surfactant. The median AI decreased after surfactant treatment; however, the decrease was not significant.

The key effector of apoptosis is a family of aspartatespecific proteases, the caspases. A model has been proposed in which caspase- 8 is activated by death receptor-mediated apoptosis, whereas caspase- 9 is activated on drug treatment, or by ultraviolet light or chemical inducers [32]. To date, neither caspase- 8 nor caspase- 9 has been investigated in human developing foetal or mechanically ventilated/oxygen-treated lungs.

Both caspases were occasionally activated in the lungs of stillborn foetuses during the saccular and canalicular stages.
To date, caspase- 9 mRNA has been demonstrated in human foetal lungs [33], which could confirm the present findings. The detection of the apoptosis-mediating surface antigen (Fas) receptor and its ligand (FasL) in bronchiolar epithelium and epithelial type II cells in developing lungs of rodents [17, 34] also support the present results. These proteins could participate in the signalling cascade leading to cleavage of caspase-8.

Surprisingly, in the lungs of preterm infants with RDS treated with mechanical ventilation at elevated oxygen concentrations, an increase in neither cleaved caspase- 8 nor -9 was detectable. However, considerably more caspase- 3 was activated compared to lung tissue sections from stillborn foetuses. In these examination groups, caspase-3-positive cells occurred in the same anatomical region and roughly the same number as TUNEL-positive cells. Obviously, a signal cascade via caspase- 3 without participation of cleaved caspases- 8 and -9 leads to cell death in the lungs of preterm infants exposed to hyperoxia. Caspase-3-mediated apoptosis in which neither caspase- 8 nor caspase- 9 is activated by cleavage was also demonstrated recently in AKR-2B cells as a consequence of serum deprivation [35].

In summary, apoptosis and proliferation are integral components of lung development in the lungs of stillborn foetuses aged 22-36 weeks of gestation. The present study has shown that the numbers of cells undergoing apoptosis and proliferation in the lungs of preterm infants with respiratory distress syndrome increase in connection with mechanical ventilation/oxygen treatment, with the duration of ventilation being an important factor. Increased apoptosis is mediated via caspase-3. Neither the apoptotic nor the proliferation index differed significantly as a result of surfactant treatment. The molecular mechanisms of apoptosis and proliferation in these mechanically ventilated/oxygen-treated lungs are still to be defined.

\section{References}

1. Barazzone C, Horowitz S, Donati YR, Rodriguez I, Piguet PF. Oxygen toxicity in mouse lung: pathways to cell death. Am J Respir Cell Mol Biol 1998; 19: 573-581.

2. McGrath-Morrow SA, Stahl J. Apoptosis in neonatal murine lung exposed to hyperoxia. Am J Respir Cell Mol Biol 2001; 25: 150-155.

3. O'Reilly MA, Staversky RJ, Stripp BR, Finkelstein JN. Exposure to hyperoxia induces p53 expression in mouse lung epithelium. Am J Respir Cell Mol Biol 1998; 18: 43-50.

4. Absher M, Makrides W, Shapiro P, Evans JN. Hyperoxia inhibits proliferation of cultured rat tracheal smooth muscle cells. Am J Physiol 1994; 267: L101-L105.

5. Hussain N, Wu F, Christian C, Kresch MJ. Hyperoxia inhibits fetal rat lung fibroblast proliferation and expression of procollagens. Am J Physiol 1997; 273: L726-L732.

6. Rancourt RC, Staversky RJ, Keng PC, O'Reilly MA. Hyperoxia inhibits proliferation of $\mathrm{Mv} 1 \mathrm{Lu}$ epithelial cells independent of TGF- $\beta$ signaling. Am J Physiol 1999; 277: L1172-L1178.

7. Baur FM, Brenner B, Götze-Speer B, Neu S, Speer CP Natural porcine surfactant (Curosurf) down-regulates mRNA of tumor necrosis factor- $\alpha$ (TNF- $\alpha)$ and TNF- $\alpha$ type II receptor in lipopolysaccharide-stimulated monocytes. Pediatr Res 1998; 44: 32-36.

8. Thomassen MJ, Antal JM, Barna BP, Divis LT, Meeker DP, Wiedemann HP. Surfactant downregulates synthesis of DNA and inflammatory mediators in normal human lung fibroblasts. Am J Physiol 1996; 270: L159-L163.

9. Hargitai B, Szabó V, Hajdú J, et al. Apoptosis in various organs of preterm infants: histopathologic study of lung, 
kidney, liver, and brain of ventilated infants. Pediatr Res 2001; 50: 110-114.

10. Crapo JD. Morphologic changes in pulmonary oxygen toxicity. Annu Rev Physiol 1986; 48: 721-731.

11. Kelleher MD, Naureckas ET, Solway J, Hershenson MB. In vivo hyperoxic exposure increases cultured lung fibroblast proliferation and c-Ha-ras expression. Am $J$ Respir Cell Mol Biol 1995; 12: 19-26.

12. Sanchez-Esteban J, Wang Y, Cicchiello LA, Rubin LP. Cyclic mechanical stretch inhibits cell proliferation and induces apoptosis in fetal rat lung fibroblasts. Am J Physiol Lung Cell Mol Physiol 2002; 282: L448-L456.

13. Smith PG, Janiga KE, Bruce MC. Strain increases airway smooth muscle cell proliferation. Am J Respir Cell Mol Biol 1994; 10: 85-90.

14. Lichnovský V, Kolár Z, Murray $\mathrm{P}$, et al. Differences in p53 and bcl-2 expression in relation to cell proliferation during the development of human embryos. J Clin Pathol Mol Pathol 1998; 51: 131-137.

15. Cass DL, Quinn TM, Yang EY, et al. Increased cell proliferation and decreased apoptosis characterize congenital cystic adenomatoid malformation of the lung. J Pediatr Surg 1998; 33: 1043-1047.

16. Kresch MJ, Christian C, Wu F, Hussain N. Ontogeny of apoptosis during lung development. Pediatr Res 1998; 43: 426-431.

17. De Paepe ME, Rubin LP, Jude C, Lesieur-Brooks AM, Mills DR, Luks FI. Fas ligand expression coincides with alveolar cell apoptosis in late-gestation fetal lung development. Am J Physiol Lung Cell Mol Physiol 2000; 279: L967-L976.

18. Weaver TE, Whitsett JA. Function and regulation of expression of pulmonary surfactant-associated proteins. Biochem J 1991; 15: 249-264.

19. Clement A, Edeas M, Chadelat K, Brody JS. Inhibition of lung epithelial cell proliferation by hyperoxia. Posttranscriptional regulation of proliferation-related genes. J Clin Invest 1992; 90: $1812-1818$

20. Shenberger JS, Dixon PS. Oxygen induces S-phase growth arrest and increases p53 and p21 $1^{\mathrm{WAF} / \mathrm{CIP} 1}$ expression in human bronchial smooth-muscle cells. Am J Respir Cell Mol Biol 1999; 21: 395-402.

21. Zhang S, Smartt H, Holgate ST, Roche WR. Growth factors secreted by bronchial epithelial cells control myofibroblast proliferation: an in vitro co-culture model of airway remodeling in asthma. Lab Invest 1999; 79: 395-405.

22. Barazzone C, Donati YR, Rochat AF, et al. Keratinocyte growth factor protects alveolar epithelium and endothelium from oxygen-induced injury in mice. Am J Pathol 1999; 154: 1479-1487.

23. Chetty A, Nielsen HC. Regulation of cell proliferation by insulin-like growth factor 1 in hyperoxia-exposed neonatal rat lung. Mol Genet Metab 2002; 75: 265-275.

24. Forkert PG, Forkert L, Farooqui M, Reynolds ES Lung injury and repair: DNA synthesis following 1,1dichloroethylene. Toxicology 1985; 36: 199-214.

25. Kazzaz JA, Xu J, Palaia TA, Mantell L, Fein AM, Horowitz S. Cellular oxygen toxicity. J Biol Chem 1996; 271: $15182-15186$.

26. Zamzami N, Marchetti P, Castedo M, et al. Sequential reduction of mitochondrial transmembrane potential and generation of reactive oxygen species in early programmed cell death. J Exp Med 1995; 182: 367-377.

27. Knaapen AM, Seiler F, Schilderman PA, et al. Neutrophils cause oxidative DNA damage in alveolar epithelial cells. Free Radic Biol Med 1999; 27: 234-240.

28. Seno $\mathrm{T}$, Inoue $\mathrm{N}$, Gao $\mathrm{D}$, et al. Involvement of NADH/ NADPH oxidase in human platelet ROS production. Thromb Res 2001; 103: 399-409.

29. Jain A, Mehta T, Auld PA, et al. Glutathione metabolism in newborns: evidence for glutathione deficiency in plasma, bronchoalveolar lavage fluid, and lymphocytes in prematures. Pediatr Pulmonol 1995; 20: 160-166.

30. Bhandari V, Maulik N, Kresch M. Hyperoxia causes an increase in antioxidant enzyme activity in adult and fetal rat type II pneumocytes. Lung 2000; 178: 53-60.

31. Kremlev SG, Umstead TM, Phelps DS. Effects of surfactant protein A and surfactant lipids on lymphocyte proliferation in vitro. Am J Physiol 1994; 267: L357-L364.

32. Sun X-M, MacFarlane M, Zhuang J, Wolf BB, Green DR, Cohen GM. Distinct caspase cascades are initiated in receptor-mediated and chemical-induced apoptosis. $J$ Biol Chem 1999; 274: 5053-5060.

33. Duan H, Orth K, Chinnaiyan AM, et al. ICE-LAP6, a novel member of the ICE/Ced-3 gene family, is activated by the cytotoxic T cell protease granzyme B. J Biol Chem 1996; 271 : 16720-16724.

34. French LE, Wilson A, Hahne M, Viard I, Tschopp J, MacDonald HR. Fas ligand expression is restricted to nonlymphoid thymic components in situ. J Immunol 1997; 159: 2196-2202.

35. Kilic M, Schäfer R, Hoppe J, Kagerhuber U. Formation of noncanonical high molecular weight caspase-3 and -6 complexes and activation of caspase-12 during serum starvation induced apoptosis in AKR-2B mouse fibroblasts. Cell Death Differ 2002; 9: 125-137. 